\title{
A geometrical theorem and the resultant of two binary forms.
}

\author{
By Professor H. F. Baker, F.R.S.
}

\section{(Received 16th November 1929. Read 17th January 1930.)}

It was proved by Salmon (Geom. of three dimensions (1882), p. 331) that the chords of the curve of intersection of two algebraic surfaces of order $m$ and $n$, which can be drawn from an arbitrary point, meet the curve upon a surface of order $(m-1)(n-1)$; it was proved by Valentiner (Acta Math. 2 (1883), p. 191), and by Noether (Berlin. Abh. (1882), Zur Grundlegung u.s.w., p. 27), that the surface of order $(m-1)(n-1)$ is a cone, with vertex at the point from which the chords are drawn; and a converse theorem was given by Halphen ( $J$. de l'école Polyt. 52 (1882), p. 106). But the proofs given by Valentiner and Noether have not the elementary character that seems desirable, Noether's proof in particular depending on the theory of the canonical series upon the curve.

In the present paper are remarked $(\$ \S 1,2,3)$ two results for the eliminant of two binary forms which, as will be seen, contain the natural continuation of Salmon's method, to prove the theorem required. It is possible these results have been remarked before. The application to the geometrical theorem is then immediate $(\S 4)$.

$$
\text { §1. Let } \begin{aligned}
f(x) & \equiv a_{0} x^{n}+\left(\begin{array}{c}
m \\
1
\end{array}\right) a_{1} x^{m-1}+\left(\begin{array}{c}
m \\
2
\end{array}\right) a_{2} x^{m-2}+\ldots \\
\phi(x) & \equiv b_{0} x^{n}+\left(\begin{array}{c}
n \\
1
\end{array}\right) b_{1} x^{n-1}+\left(\begin{array}{c}
n \\
2
\end{array}\right) b_{2} x^{n-2}+\ldots
\end{aligned}
$$

be two binary forms, and for simplicity of printing put at first $p_{i}$ and $q_{i}$ for the coefficients of $x^{m-i}, x^{n-i}$ in these; denote by $\Delta_{k}$ the determinant of $m+n-2 k$ rows and columns

$$
\Delta_{k} \equiv\left|\begin{array}{cccc}
p_{0} & p_{1} & p_{2} & \ldots \\
0 & p_{0} & p_{1} & \ldots \\
0 & 0 & p_{0} & \ldots \\
\ldots & \ldots & \ldots \\
q_{0} & q_{1} & q_{2} & \ldots \\
0 & q_{0} & q_{1} & \ldots \\
\ldots & \ldots & \ldots
\end{array}\right|,
$$

wherein there is first a set of $n-k$ rows containing the elements $p_{0}, p_{1}, \ldots$ in order, beginning in turn in the first, second, ... 
columns, then a set of $m-k$ rows containing the elements $q_{0}, q_{1}, \ldots$ in order, beginning in turn in the first, second, ... columns. Then it is known that the vanishing of the determinants

$$
\Delta_{0}, \Delta_{1}, \Delta_{2}, \ldots, \Delta_{s-1}
$$

is the necessary and sufficient condition that the original binary forms have as common factor a polynomial of order $s$ ( $C f$. Scheibner, Leipzig. Ber. 40 (1880), p. 1; Kronecker, Werke 2 (1881), p. 115 ; Netto, Algebra, 1 (1896), p. 157). What I propose to prove here is that the determinant $\Delta_{k}$ satisfies the equation

$\left(a_{0} \frac{\partial}{\partial a_{1}}+2 a_{1} \frac{\partial}{\partial a_{2}}+\ldots+m a_{m-1} \frac{\partial}{\partial a_{m}}+b_{0} \frac{\partial}{\partial b_{1}}+2 b_{1} \frac{\partial}{\partial b_{2}}+\ldots+n a_{n-1} \frac{\partial}{\partial a_{n}}\right) \Delta_{k}=0$

This, it is known, is the same as saying that $\Delta_{k}$ is unaffected if we write therein for $a_{r}, b_{s}$ respectively the elements

$$
\begin{aligned}
& \bar{a}_{r}=a_{r}+r a_{r-1} \theta+\left(\begin{array}{l}
r \\
2
\end{array}\right) a_{r-2} \theta^{2}+\ldots+a_{0} \theta^{r}, \\
& \bar{b}_{s}=b_{s}+s a_{s-1} \theta+\left(\begin{array}{c}
s \\
2
\end{array}\right) a_{s-2} \theta^{2}+\ldots+b_{0} \theta^{s},
\end{aligned}
$$

wherein $\theta$ is arbitrary; putting $x=\xi+\theta$, these are the coefficients of the powers of $\xi$ in the expansions

$$
\begin{aligned}
& f(\xi+\theta)=\bar{a}_{0} \xi^{m}+\left(\begin{array}{c}
m \\
1
\end{array}\right) \bar{a}_{1} \xi^{m-1}+\ldots+\bar{a}_{m}, \equiv f, \text { say }, \\
& \phi(\xi+\theta)=\bar{b}_{0} \xi^{n}+\left(\begin{array}{c}
n \\
1
\end{array}\right) \bar{b}_{1} \xi^{n-1}+\ldots+\bar{b}_{n}, \equiv \bar{\phi}, \text { say. }
\end{aligned}
$$

If from the determinant $\Delta_{0}$ we form a determinant of $m+n+1$ rows and columns by supplying a new first row containing unity in the first and $(m+n+1)^{\text {th }}$ columns, and otherwise zeros, and supplying a new first column consisting of the elements

$$
1, x^{n-1} f, x^{n-2} f, \ldots, f, x^{m-1} \phi, x^{m-2} \phi, \ldots, \phi
$$

in order, this new determinant is identically zero, as we see by subtracting from the first column the other columns multiplied in order respectively by $x^{m+n-1}, x^{m+n-2}, \ldots, 1$. Thus by expanding this new determinant according to the elements of the first column we have the identity

$$
\begin{aligned}
\Delta_{0}=\left(b_{0}^{\prime} x^{n-1}+\left(\begin{array}{c}
n-1 \\
1
\end{array}\right)\right. & \left.b_{1}^{\prime} x^{n-2}+\ldots+b_{n-1}^{\prime}\right) f \\
& +\left(a_{0}^{\prime} x^{m-1}+\left(\begin{array}{c}
m-1 \\
1
\end{array}\right) a_{1}^{\prime} x^{n-2}+\ldots+a_{m-1}^{\prime}\right) \phi
\end{aligned}
$$

where $a_{0}^{\prime}, \ldots, a_{m-1}^{\prime}, b_{0}^{\prime}, \ldots, b_{n-1}^{\prime}$ are certain minors of $\Delta_{0}$, obtained by 
omitting the last column, and one row. And it is important for our purpose to note, what is seen at once on inspection, that, save for a \pm sign

$$
a_{0}^{\prime}=a_{0} \Delta_{1}, \quad b_{0}^{\prime}=b_{0} \Delta_{1} .
$$

Now we might have proceeded in precisely the same way with the polynomials in $\xi$ denoted by $\bar{f}$ and $\bar{\phi}$, and obtained an identity.

$$
\bar{\Delta}_{0}=\left(\bar{b}_{0}^{\prime} \xi^{n-1}+\ldots+b_{n-1}^{\prime}\right) \bar{f}+\left({\overline{a^{\prime}}}_{0} \xi^{n-1}+\ldots+\bar{a}_{n-1}^{\prime}\right) \bar{\phi} ;
$$

but $\bar{f}, \bar{\phi}$ are respectively $f$ and $\phi$, differently expressed; thus from these two identities, in the general case when $f$ and $\phi$ have no common linear factor, we can infer, since $\bar{\Delta}_{0}$ contains the term $\bar{a}_{0}{ }^{n} \bar{b}_{n}{ }^{m}$, or $a_{0}{ }^{n} \bar{b}_{n}{ }^{m}$, which contains the term $a_{0}{ }^{n} b_{n}{ }^{m}$, that $\bar{\Delta}_{0}=\Delta_{0}$ and that

$$
{\overline{a^{\prime}}}_{0} \xi^{m-1}+\ldots+{\overline{a_{m-1}^{\prime}}}_{m},{\overline{b^{\prime}}}_{0} \xi^{n-1}+\ldots+b_{n-1}^{\prime}
$$

are respectively the same as

$$
a_{0}^{\prime} x^{m-1}+\ldots+a_{m-1}^{\prime}, b_{0}^{\prime} x^{n-1}+\ldots+b_{n-1}^{\prime} \text {. }
$$

This is clear also from the fact that $\Delta_{0}=0$ is, as the first identity shews, the necessary and sufficient condition for $f, \phi$ to have a common linear factor, and is hence, as is known, save for proper powers of $a_{0}, b_{0}$, the product of every difference of a root of $\phi=0$ and a root of $f=0$, and is therefore unaltered by writing $\xi+\theta$ for $x$ in $f$ and $\phi$.

From the identity of

$$
a_{0}^{\prime}(\xi+\theta)^{m-1}+\ldots+a_{m-1}^{\prime}, \bar{a}_{0}^{\prime} \xi^{m-1}+\ldots+\bar{a}_{m-1}^{\prime}
$$

however, we infer that $\bar{a}_{0}^{\prime}=a_{0}^{\prime}$, and hence

and hence $\overline{\Delta_{1}}=\Delta_{1}$

$$
a_{0} \bar{\Delta}_{1}=a_{0} \Delta_{1}
$$

It is thus shewn that $\Delta_{1}$ is unchanged when $a_{0}, \bar{a}_{1}, \bar{a}_{2}, \ldots$ are written in place of $a_{0}, a_{1}, a_{2}, \ldots$.

But from the identity of these two polynomials of order $m-1$, we also infer that

with similar equations for $\bar{b}_{s}^{\prime}$.

$$
\overline{a_{r}^{\prime}}=a_{r}^{\prime}+r a_{r-1}^{\prime} \theta+\left(\begin{array}{l}
r \\
2
\end{array}\right) a_{r-2}^{\prime} \theta^{2}+\ldots
$$

2. Now from the two forms

$$
\begin{aligned}
& a_{0}^{\prime} x^{m-1}+\left(\begin{array}{c}
m-1 \\
1
\end{array}\right) a_{1}^{\prime} x^{n-2}+\ldots \\
& b_{0}^{\prime} x^{n-1}+\left(\begin{array}{c}
n-1 \\
1
\end{array}\right) b_{1}^{\prime} x^{n-2}+\ldots
\end{aligned}
$$

form the determinant, $\Delta_{0}^{\prime}$, of $m+n-2$ rows and columns, just 
as $\Delta_{0}$ was formed from the two original forms $f$ and $\phi$. We can prove that, independently of the condition $\Delta_{0}=0$, this $\Delta_{0}^{\prime}$ is identically a power of $\Delta_{1}$, and hence, counting the dimensions involved, that

$$
\Delta_{0}^{\prime}=\epsilon \Delta_{1}^{m+n-1}
$$

where $\epsilon$ is a \pm sign easy to determine.

For suppose $\Delta_{0}^{\prime}$ is zero, but $\Delta_{0}$ is not zero. Then, the two equations

$$
a_{0}^{\prime} x^{m-1}+\ldots=0, b_{0}^{\prime} x^{n-1}+\ldots=0,
$$

by the well known property of the resultant, quoted above, have a common root. From the original identity

$$
\Delta_{0}=\left(b_{0}^{\prime} x^{n-1}+\ldots\right) f+\left(a_{0}^{\prime} x^{m-1}+\ldots\right) \phi,
$$

however, this is impossible for a finite value of $x$, as $\Delta_{0} \neq 0$, The only possibility is thus an infinite root, namely $a_{0}^{\prime}=0, b_{0}^{\prime}=0$. These however are $a_{0} \Delta_{1}=0$ and $b_{0} \Delta_{1}=0$. Wherefore the equation $\Delta^{\prime}{ }_{0}=0$ involves $\Delta_{1}=0$; and this can only be if $\Delta_{0}^{\prime}$ is a power of $\Delta_{1}$.

The result seems natural, and interesting, but may have been remarked before. A particular case was set in a college examination paper of June 1894.

3. Now consider the identity

$$
\Delta_{0}^{\prime}=\left(b_{0}^{\prime \prime} x^{n-2}+\ldots+b_{n-2}^{\prime \prime}\right) f^{\prime}+\left(a_{0}^{\prime \prime} x^{m-2}+\ldots+a_{m-2}^{\prime \prime}\right) \phi^{\prime}
$$

for the two forms

$$
f^{\prime}=a_{0}^{\prime} x^{m-1}+\ldots+a_{m-1}^{\prime}, \phi^{\prime}=b_{0}^{\prime} x^{n-1}+\ldots+b_{n-1}^{\prime},
$$

analogous to the original identity for $f$ and $\phi$. As we have proved (in $\S 1$ ) that the determinant $\Delta_{1}$, obtained from $\Delta_{0}$ by omitting two rows and columns, is unaffected by writing in $\Delta_{1}$ for $a_{r}, b_{s}$ respectively

$$
a_{r}+r a_{r-1} \theta+\ldots, b_{s}+s a_{s-1} \theta+\ldots,
$$

so it is true that the determinant $\Delta_{1}^{\prime}$, of $m+n-4$ rows and columns, obtained from $\Delta_{0}^{\prime}$ by omitting two rows and columns, is unaffected by a similar substitution of

$$
a_{r}^{\prime}+r a_{r-1}^{\prime} \theta+\ldots, b_{s}^{\prime}+s a_{s-1}^{\prime} \theta+\ldots
$$

for $a_{r}^{\prime}, b_{s}^{\prime}$ : and we have shewn that these arise by the original substitutions for $a_{r}$ and $b_{s}$. But, also by what we shewed, save for a \pm sign,

$$
a_{0}^{\prime \prime}=a_{0}^{\prime} \Delta_{1}^{\prime}, \quad b_{0}^{\prime \prime}=b_{0}^{\prime} \Delta_{1}^{\prime},
$$

and, by an argument employed above, writing $\xi+\theta$ for $x$ in the identity

$$
\Delta_{0}^{\prime}=\left(b_{0}^{\prime \prime} x^{n-2}+\ldots\right) f^{\prime}+\left(a_{0}^{\prime \prime} x^{m-2}+\ldots\right) \phi^{\prime}
$$


both $a_{0}^{\prime \prime}, b_{0}^{\prime \prime}$ are unaffected by the substitution of $a_{r}^{\prime}+r a_{r-1}^{\prime} \theta+\ldots$, $b_{s}^{\prime}+s a_{s-1}^{\prime} \theta+\ldots$ for $a_{r}^{\prime}, b_{s}^{\prime}$, and therefore by the original substitution. of $\bar{a}_{r}, \bar{b}_{s}$ for $a_{r}, b_{s}$. Whence it is clear that $\Delta^{\prime}{ }_{1}$ is unaffected thereby.

But $\Delta_{1}^{\prime}=0$ is the condition which must be added to $\Delta_{0}^{\prime}=0$, (that is to $\Delta_{1}=0$, by what is shewn in $\S 2$ ), in order that $f^{\prime}$ and $\phi^{\prime}$ may have a common quadratic factor, that is $\Delta_{0}=0, \Delta_{1}=0, \Delta^{\prime}{ }_{1}=0$ are the conditions that $f, \phi$ may have a common cubic factor. Wherefore $\Delta_{1}^{\prime}=0$ is the same when $\Delta_{0}=0, \Delta_{1}=0$ as $\Delta_{2}=0$.

Whence we infer that $\Delta_{2}$ is likewise unaffected by the same substitution of $a_{r}+a_{r-1} \theta+\ldots, b_{s}+s b_{s-1} \theta+\ldots$ for $a_{r}, b_{s}$.

And the argument may be continued.

For an example take $m=4, n=3$. Then $\Delta_{2}$ is

$$
\begin{array}{rrr}
a_{0} & 4 a_{1} & 6 a_{2} \\
b_{0} & 3 b_{1} & 3 b_{2} \\
& b_{0} & 3 b_{1}
\end{array}
$$

or $a_{0}\left(9 b_{1}{ }^{2}-3 b^{0} b_{2}\right)-12 a_{1} b_{0} b_{1}+6 b_{0}^{2} a_{2}$

which by the differential operator of $\S 1$ becomes the identically vanishing expression.

$$
a_{0}\left(18 b_{0} b_{1}-6 b_{0} b_{1}\right)-12 a_{0} b_{0} b_{1}-12 a_{1} b_{0}^{2}+12 b_{0}^{2} a_{1} .
$$

In general $\Delta_{k}$ is the first coefficient in a covariant of $f$ and $\phi$ of order $k(m+n-2 k)$. For example for $f=a_{x}{ }^{3}, \phi=b_{x}{ }^{2}$ this covariant for $k=1$ is

$$
-\frac{1}{2}\left(b b^{\prime}\right)^{2} a_{x}{ }^{3}+3(a b)\left(a b^{\prime}\right) a_{x} b_{x} b_{x}^{\prime},
$$

where $b, b^{\prime}$ are equivalent symbols.

$\S 4$. Now suppose that

$$
f_{x}{ }^{n}=0, \phi_{x}{ }^{n}=0
$$

are the equations of two surfaces, in ordinary space, of orders $m, n$. Taking a fixed point $(\xi)$, and a point $(x)$, substitute herein $\lambda \xi+x, \lambda \eta+y, \lambda \zeta+z, \lambda \tau+t$, so obtaining the equations

$$
\begin{array}{ll}
\lambda^{m} f_{\xi} m+\left(\begin{array}{c}
m \\
1
\end{array}\right) \lambda^{m-1} f_{\xi}^{m-1} f_{x}+\ldots & +f_{x}^{m}=0 \\
\lambda^{n} \phi_{\xi}^{n}+\left(\begin{array}{c}
n \\
1
\end{array}\right) \lambda^{n-1} \phi_{\xi}^{n-1} \phi_{x}+\ldots & +\phi_{x}^{n}=0
\end{array}
$$

which we may identify with our original equations ( $\lambda$ being put for $x$ )

$$
\begin{array}{ll}
a_{0} \lambda^{m}+\left(\begin{array}{c}
m \\
1
\end{array}\right) a_{1} \lambda^{m-1}+\ldots & +a_{m}=0 \\
b_{0} \lambda^{n}+\left(\begin{array}{c}
n \\
1
\end{array}\right) b_{1} \lambda^{n-1}+\ldots & +b_{n}=0 .
\end{array}
$$


The condition that these two equations in $\lambda$ should have a common root is $\Delta_{0}=0$. This however, when $(x)$ are current coordinates, represents a cone. For if in $(\lambda \xi+x, \ldots)$ we write $(x+\theta \xi, \ldots, t+\theta \tau)$ for $(x, \ldots, t)$, this will be equivalent to considering the equations

$$
\bar{a}_{0} \lambda^{m}+\left(\begin{array}{c}
m \\
1
\end{array}\right) \bar{a}_{1} \lambda^{m-1}+\ldots=0, \quad \bar{b}_{0} \lambda^{n}+\ldots=0
$$

obtained from the original two by writing $\lambda+\theta$ in place of $\lambda$; and the equation $\bar{\Delta}_{0}=0$ obtained from these is the same as $\Delta_{0}=0$. The locus $\Delta_{0}=0$ is thus unaffected by writing $(x+\theta \xi, \ldots, t+\theta \tau)$ for $(x, \ldots, t)$, and represents a cone with vertex at $(\xi)$. It is the cone to the common curve of the two surfaces.

The equation $\Delta_{1}=0$ likewise represents a cone. And as $\Delta_{0}=0$, $\Delta_{1}=0$ are the conditions for a pair of common roots of the two $\lambda$ equations, it follows that $\Delta_{1}=0$ is a cone containing the chords from $(\xi)$ of the curve of intersection of the surfaces. The determinant $\Delta_{1}$ is of weight $(m-1)(n-1)$; this is then the order of the cone $\Delta_{1}$.

The argument is not confined to the case of surfaces in threefold space. For instance, in space of any dimension, the lines from

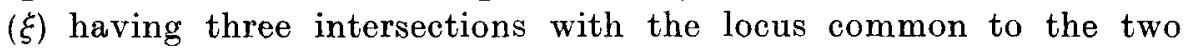
original primals $f=0, \phi=0$ lie upon the three cones $\Delta=0, \Delta_{1}=0$, $\Delta_{2}=0$.

Remark. The condition, in the geometrical problem, that the lines joining $(x),(\xi)$ may meet the two surfaces $f=0, \phi=0$ in the same two points may equally be expressed by $\nabla_{0}=0, \nabla_{1}=0$, where $\nabla_{0}$ is the same as $\Delta_{0}$ with the coofficients $a_{0} \ldots a_{m}, b_{0}, \ldots b_{n}$ in the two $\lambda$ equations taken in the reverse order, namely $a_{r} b_{s}$ replaced by $a_{m-r}, b_{n-s}$, and $\nabla_{1}$ is formed from $\nabla_{0}$ as was $\Delta_{1}$ from $\Delta_{0}$. And, evidently, if $(x)$ be any point common to the two loci $\nabla_{0}=0, \nabla_{1}=0$, so also is $(x+\theta \xi, \ldots, t+\theta \tau)$, whatever $\theta$ may be-For if we write $(x+\theta \xi, \ldots)$ for $(x, \ldots)$ in $(\lambda \xi+x, \ldots)$ this is only equivalent to putting originally $((\lambda+\theta) \xi+x, \ldots)$. The locus $\nabla_{0}$ is identically the same as $\Delta_{0}$, and represents a cone. Thus $\nabla_{1}=0$ represents a surface containing the chords from $(\xi)$ to the curve common to $f=0, \phi=0$. The determinant $\nabla_{1}$ contains the term $a_{m}^{n-1} b_{1}^{m-1}$, and is isobaricThus $\nabla_{1}=0$ is a surface of order $m n-1$ containing the chords of the curve which can be drawn from $(\xi)$. 